\title{
Interpolation Methods for Stochastic Processes Spaces
}

\author{
E. Nursultanov ${ }^{1}$ and T. Aubakirov ${ }^{2}$ \\ ${ }^{1}$ Lomonosov Moscow State University (Kazakh Branch) and Gumilyov Eurasian National University, Munatpasova 7, \\ Astana 010010, Kazakhstan \\ ${ }^{2}$ Autonomous Organization of Education Nazarbayev Intellectual Schools, Astana 010010, Kazakhstan \\ Correspondence should be addressed to E. Nursultanov; er-nurs@yandex.ru
}

Received 2 September 2013; Revised 4 November 2013; Accepted 18 November 2013

Academic Editor: Henryk Hudzik

Copyright (C) 2013 E. Nursultanov and T. Aubakirov. This is an open access article distributed under the Creative Commons Attribution License, which permits unrestricted use, distribution, and reproduction in any medium, provided the original work is properly cited.

\begin{abstract}
The scales of classes of stochastic processes are introduced. New interpolation theorems and boundedness of some transforms of stochastic processes are proved. Interpolation method for generously monotonous processes is entered. Conditions and statements of interpolation theorems concern the fixed stochastic process, which differs from the classical results.
\end{abstract}

\section{Introduction}

Interpolation methods of functional spaces are one of the basic tools to get inequalities in parametrical spaces. These methods are widely applied in the theory of stochastic processes (see [1-5] and other).

In this paper classes of stochastic processes are considered, which, in some sense, are analogues of the net spaces which were investigated in [6-8].

Assume that $(\Omega, \mathfrak{G}, P)$ is a complete probability space. A family $G=\left\{\mathfrak{G}_{n}\right\}_{n \geqslant 1}$ of $\sigma$-algebras $\mathfrak{G}_{n}$ such that $\mathfrak{G}_{1} \subseteq \cdots \subseteq$ $\mathfrak{G}_{n} \subseteq \cdots \subseteq \mathfrak{G}$ is called a filtration.

Let $G$ be a filtration, a sequence $\left\{X_{n}\right\}_{n \geqslant 1}$ of random variables $X_{n}$ measurable function with respect to the $\sigma$ algebra $\mathfrak{G}_{n}$. Then we say that the set $X=\left(X_{n}, \mathfrak{S}_{n}\right)_{n \geqslant 1}$ is a stochastic process.

Let $F=\left\{\mathfrak{F}_{n}\right\}_{n \geqslant 1}$ be a system of sets satisfying the condition $\mathfrak{F}_{1} \subseteq \cdots \subseteq \mathfrak{F}_{n} \subseteq \cdots \subseteq \mathfrak{G}$. We say that a stochastic process $X=\left(X_{n}, \mathfrak{G}_{n}\right)_{n \geqslant 1}$ is defined on a system $F=\left\{\mathfrak{\mho}_{n}\right\}_{n \geqslant 1}$ if $\mathfrak{F}_{n} \subset \mathfrak{G}_{n}, n \in \mathbb{N}$. For a stochastic process $X$, which is defined on a system $F=\left\{\mathfrak{F}_{n}\right\}_{n \geqslant 1}$, we define the sequence of numbers $\bar{X}(F)=\left\{\bar{X}_{n}(F)\right\}_{n}$, where

$$
\bar{X}_{k}(F)=\sup _{A \in \mathfrak{F}_{k}, P(A)>0} \frac{1}{P(A)}\left|\int_{A} X_{k} P(d \omega)\right| .
$$

We call this sequence a majorant of a process $X$ on a system of sets $F$.

Let us give some examples of a choice of a system of sets $F=\left\{\mathfrak{F}_{n}\right\}_{n \geqslant 1}: \mathfrak{F}_{n}=\Omega$, in this case the sequence $\bar{X}(F)=$ $\left\{\bar{X}_{n}(F)\right\}_{n}$ is a sequence of averages of a process $X=\left\{X_{n}\right\}$; for $\mathfrak{F}_{n}=\mathfrak{G}_{n-1}$ it is a majorant of sequence of conditional averages $M\left(X_{n} \mid G_{n-1}\right)$; and for $\mathfrak{F}_{n}=\mathfrak{G}_{n}$ it is a majorant of a process $X=\left\{X_{n}\right\}$. The following cases are interesting: (1) $\mathfrak{F}_{n}=\mathfrak{G S}_{n \wedge \tau}$, where $\tau=\tau(\omega)$ is the fixed stopping time, and $\mathfrak{G}_{n \wedge \tau}=\left\{A \in \mathfrak{G}_{n}: A \cap\{\tau=n\} \in \mathfrak{G}_{n}\right\} ;(2) \mathfrak{F}_{n}=\mathfrak{G}_{n \wedge \tau_{n}}$, where $\tau_{n}=\tau_{n}(\omega)$, and $n \in \mathbb{N}$ is the sequence of the stopping times.

We consider the classes of stochastic processes defined on $F$, which characterize the speed of convergence of sequence $\left\{\bar{X}_{n}(F) / n\right\}_{n}$ to zero.

By $N_{p, q}(F), 0<p<\infty, 0<q \leqslant \infty$ we denote the set of all stochastic processes $X$, defined on $F$ for which

$$
\|X\|_{N_{p, q}(F)}=\left(\sum_{k=1}^{\infty} k^{-1-(q / p)} \bar{X}_{k}^{q}\right)^{1 / q}<\infty
$$

if $0<q<\infty$ and

$$
\|X\|_{N_{p, \infty}(F)}=\sup _{k} k^{-1 / p} \bar{X}_{k}<\infty
$$

if $q=\infty$ 
Let us denote

$$
\begin{aligned}
& N_{p}^{\alpha, q}(F) \\
& =\left\{X=\left(X_{n}, \mathfrak{\mho}_{n}\right)_{n \geqslant 1}:\left(\sum_{k=0}^{\infty}\left(2^{\alpha k} \overline{\Delta X_{k}}\right)^{q}\right)^{1 / q}<\infty\right\}
\end{aligned}
$$

for $0<q<\infty$, and

$$
N_{p}^{\alpha, \infty}(F)=\left\{X=\left(X_{n}, \mathfrak{\mho}_{n}\right)_{n \geqslant 1}: \sup _{k} 2^{\alpha k} \overline{\Delta X_{k}}<\infty\right\},
$$

for $q=\infty$, where

$$
\begin{aligned}
& \overline{\Delta X_{k}} \\
& =\sup _{A \in \mathfrak{G}, P(A)>0} \frac{1}{(P(A))^{1-(1 / p)}}\left|\int_{A}\left(X_{2^{k}}-X_{2^{k-1}}\right) P(d \omega)\right| .
\end{aligned}
$$

We consider that

$$
X_{\alpha}(\omega)= \begin{cases}X_{[\alpha]}(\omega) & \text { if } \alpha \geqslant 1 \\ 0 & \text { if } \alpha<1,\end{cases}
$$

where $[\alpha]$ is the integer part of the number $\alpha$. In particular $X_{1 / 2}(\omega) \equiv 0$.

A random variable $\tau$, which takes values in the set $(1,2, \ldots, \infty)$, is called the Markov time of the filtration $G=$ $\left\{\mathfrak{G}_{n}\right\}_{n \geqslant 1}$, if $\{\omega: \tau(\omega)=n\} \in \mathfrak{G}_{n}$ for any $n \in \mathbb{N}$. The Markov time $\tau$, for which $\tau(\omega)<\infty$ (a.p.) [9], is called the stopping time.

Let $X=\left(X_{n}, \mathfrak{G}_{n}\right)_{n \geqslant 1}$ be a stochastic process and $\tau$ be the Markov time. By $X^{\tau}$ we denote the stopped process $X^{\tau}=$ $\left(X_{n \wedge \tau}, \mathfrak{G}_{n}\right)$, where $X_{n \wedge \tau}(\omega)=\sum_{m=1}^{n-1} X_{m} \chi_{\tau=m}(\omega)+X_{n} \chi_{\tau \geqslant n}(\omega)$ and $\chi_{A}(\omega)$ is the characteristic function of the set $A$.

We assume also that

$$
X^{\alpha}= \begin{cases}X^{[\alpha]} & \text { if } \alpha \geqslant 1 \\ 0=\left(0, \mathfrak{G}_{n}\right)_{n \leqslant 1} & \text { if } \alpha<1,\end{cases}
$$

$\sum_{k=\alpha}^{\beta} b_{k}:=\sum_{k=[\alpha]}^{[\beta]} b_{k}$.

The $N_{p}^{\alpha, q}(F)$ spaces are spaces of converging stochastic processes, where parameters $\alpha, q$, and $p$ characterize the speed and the metrics, in which a given process converges.

In this paper we prove a Marcinkiewicz-type interpolation theorem for the introduced space. An interpolation method, essentially related to the properties of the Markov stopping times, is introduced. In the last paragraph the given interpolation method is applied to Besov type space with variable approximation properties. Part of the results were announced in [10].

We write $A \lesssim B$ (or $A \gtrsim B$ ) if $A \leqslant c B$ (or $c A \geqslant$ $B$ ) for some positive constant $c$ independent of appropriate quantities involved in the expressions $A$ and $B$. Notation $A=$ $B$ means that $A \lesssim B$ and $A \gtrsim B$.

\section{Properties of the Spaces $N_{p, q}(F)$ and $N_{p}^{\alpha, q}(F)$}

We say [9] that stochastic process $\left(X_{n}, \mathfrak{G}_{n}\right)_{n \geqslant 1}$ is a martingale if for every $n \in \mathbb{N}$ the following conditions hold:
(1) $E\left|X_{n}\right|<\infty$; (2) $E\left(X_{n+1} \mid \mathfrak{G}_{n}\right)=X_{n}$ (a.p.). If instead of property (2) it is assumed that $E\left(X_{n+1} \mid \mathfrak{G}_{n}\right) \geqslant X_{n}\left(E\left(X_{n+1} \mid\right.\right.$ $\left.\left(\mathfrak{G}_{n}\right) \leqslant X_{n}\right)$, then we say that a process $X=\left(X_{n}, \mathfrak{G}_{n}\right)_{n=1}^{\infty}$ is a submartingale (supermartingale).

Definition 1. Let $F=\left\{\mathfrak{F}_{n}\right\}_{n}$ be a fixed system of sets, $X=$ $\left(X_{n}, \mathfrak{G}_{n}\right)_{n}$ be a stochastic process defined on $F$. We say that a process $X$ belongs to the class $W(F)$ if there exists a constant $c$ such that for every $k \leqslant m$ and for every $A \in \mathfrak{F}_{k}$

$$
\left|\int_{A} X_{k} P(d \omega)\right| \leqslant C\left|\int_{A} X_{m} P(d \omega)\right| \text {. }
$$

This inequality implies that $\bar{X}_{k}(F) \leqslant c \bar{X}_{m}(F)$ for every $k \leqslant m$. The class $W(F)$ contains martingales, nonnegative submartingales, and nonpositive supermartingales. The stochastic process from $W(F)$ we call generalized monotone.

Lemma 2. Let $\alpha>0,0<q \leqslant \infty$, and $1<p<\infty$. If $X=$ $\left(X_{n}, \mathfrak{G}_{n}\right)_{n \geqslant 1} \in N_{p}^{\alpha, q}(G) \cap W(G)$, then there exists a random variable $X_{\infty}$ such that $X_{n} \underset{n \rightarrow \infty}{\rightarrow} X_{\infty}$ (a.p.).

Proof. Let $L_{p, \infty}(\Omega)$ be the Marcinkiewicz-Lorentz space and $2^{\nu-1} \leqslant n<2^{\nu}$. Using the equivalent norm of $L_{p, \infty}(\Omega)$ spaces (see [6]) and measurability of function $X_{n}$ with respect to $\sigma$ algebra $\mathfrak{G}_{n}$, we get the following:

$$
\begin{aligned}
& \left\|X_{n}\right\|_{L_{p, \infty}[0,1]} \\
& \quad=\sup _{A \in \mathfrak{G}, P(A)>0} \frac{1}{(P(A))^{1 / p^{\prime}}}\left|\int_{A} X_{n} P(d \omega)\right| \\
& =\sup _{A \in \mathfrak{G}_{n}, P(A)>0} \frac{1}{(P(A))^{1 / p^{\prime}}}\left|\int_{A} X_{n} P(d \omega)\right| \\
& \leqslant C \sup _{A \in \mathfrak{G}_{2^{v}}, P(A)>0} \frac{1}{(P(A))^{1 / p^{\prime}}}\left|\int_{A} X_{2^{v}} P(d \omega)\right| \\
& \leqslant C \sum_{k=0}^{v} \sup _{A \in \mathfrak{G}_{2^{k}}, P(A)>0} \frac{1}{(P(A))^{1 / p^{\prime}}} \\
& \quad \times\left|\int_{A}\left(X_{2^{k}}-X_{2^{k-1}}\right) P(d \omega)\right| \\
& \leqslant C\|X\|_{N_{p}^{0,1}(G)} .
\end{aligned}
$$

Taking into account that $N_{p}^{\alpha, q}(G) \hookrightarrow N_{p}^{0,1}(G)$, for $\alpha>0$, we have $\left\|X_{n}\right\|_{L_{p, \infty}[0,1]} \leqslant c\|X\|_{N_{p}^{\alpha, q}(G)}$.

But $M\left|X_{n}\right| \leqslant c\left\|X_{n}\right\|_{L_{p, \infty}[0,1]}$, therefore by the Doob theorem ([11]), the process $X_{n}$ converges almost surely.

Lemma 3. Let $\alpha>0,0<q \leqslant \infty, 1<p<\infty$, and $X=$ $\left(X_{n}, \mathfrak{G}_{n}\right)_{n \geqslant 1} \in W(G)$. Then

$$
\|X\|_{N_{p}^{\alpha, q}(G)}=\left(\sum_{k=0}^{\infty}\left(2^{\alpha k \widetilde{X_{k}}}\right)^{q}\right)^{1 / q}
$$

where

$$
\widetilde{X_{k}}=\sup _{A \in \mathfrak{G}, P(A)>0} \frac{1}{(P(A))^{1 / p^{\prime}}}\left|\int_{A}\left(X_{\infty}-X_{2^{k-1}}\right) P(d \omega)\right| .
$$


Proof. The existence of $X_{\infty}$ follows from Lemma 2. Further, we have

$$
\begin{aligned}
\widetilde{X_{k}} & =\sup _{P(A)>0} \frac{1}{(P(A))^{1 / p^{\prime}}}\left|\int_{A} \sum_{m=k}^{\infty}\left(X_{2^{m}}-X_{2^{m-1}}\right) P(d \omega)\right| \\
& \leqslant \sum_{m=k}^{\infty} \sup _{A \in \mathfrak{S}_{2^{m}}} \frac{1}{(P(A))^{1 / p^{\prime}}}\left|\int_{A}\left(X_{2^{m}}-X_{2^{m-1}}\right) P(d \omega)\right| \\
& =\sum_{m=k}^{\infty} \overline{\Delta X}_{m} .
\end{aligned}
$$

Therefore, using Lemma 8, we obtain

$$
\begin{aligned}
\left(\sum_{k=0}^{\infty}\left(2^{\alpha k} \widetilde{X_{k}}\right)^{q}\right)^{1 / q} & \leqslant\left(\sum_{k=0}^{\infty}\left(2^{\alpha k} \sum_{m=k}^{\infty} \overline{\Delta X}_{m}\right)^{q}\right)^{1 / q} \\
& \leqslant\left(\sum_{m=0}^{\infty} 2^{m \alpha q} \overline{\Delta X}_{m}^{q}\right)^{1 / q} \\
& =\|X\|_{N_{p}^{\alpha, q}(G)} .
\end{aligned}
$$

The reverse inequality follows from the expression:

$$
\begin{aligned}
\overline{\Delta X}_{k}= & \sup _{P(A)>0} \frac{1}{(P(A))^{1 / p^{\prime}}}\left|\int_{A}\left(X_{2^{k}}-X_{2^{k-1}}\right) P(d \omega)\right| \\
\leqslant & \sup _{P(A)>0} \frac{1}{(P(A))^{1 / p^{\prime}}}\left|\int_{A}\left(X_{2^{k}}-X_{\infty}\right) P(d \omega)\right| \\
& +\sup _{P(A)>0} \frac{1}{(P(A))^{1 / p^{\prime}}}\left|\int_{A}\left(X_{\infty}-X_{2^{k-1}}\right) P(d \omega)\right| .
\end{aligned}
$$

The lemma is proved.

Lemma 4. Let $X \in W(F)$. Then

(1) for $0<q \leqslant q_{1} \leqslant \infty$,

$$
\begin{gathered}
\|X\|_{N_{p, q_{1}}(F)} \leqslant c_{p, q, q_{1}}\|X\|_{N_{p, q}(F)}, \\
\|X\|_{N_{p}^{\alpha, q_{1}}(F)} \leqslant c_{\alpha, p, q, q_{1}}\|X\|_{N_{p}^{\alpha, q}(F)},
\end{gathered}
$$

(2) for $\varepsilon>0,0<q, q_{1} \leqslant \infty$,

$$
\begin{gathered}
\|X\|_{N_{p, q_{1}}(F)} \leqslant c_{p, q, q_{1}}\|X\|_{N_{p+\varepsilon, q}(F)}, \\
\|X\|_{N_{p}^{\alpha, q_{1}}(F)} \leqslant c_{\alpha, p, q, q_{1}}\|X\|_{N_{p}^{\alpha+\varepsilon, q}(F)},
\end{gathered}
$$

where $c_{p, q, q_{1}}, c_{\alpha, p, q, q_{1}}>0$ depend only on the indicated parameters.

Proof. Let us prove inequalities (16), (18). The proof of inequalities (17), (19) is similar. Let $\varepsilon>0$. By Minkowski's inequality and by the generalized monotonicity of a process $X=\left(X_{n}, \mathfrak{G}_{n}\right)_{n \geqslant 1}$ we get the following:

$$
\begin{aligned}
\|X\|_{N_{p, q_{1}}(F)} & =\left(\sum_{k=1}^{\infty} k^{\varepsilon q_{1}-1} k^{-\varepsilon q_{1}-\left(q_{1} / p\right)} \bar{X}_{k}^{q_{1}}\right)^{1 / q_{1}} \\
& \lesssim\left(\sum_{k=1}^{\infty} k^{\varepsilon q_{1}-1}\left(\sum_{r=k}^{\infty} r^{-q(\varepsilon+(1 / p))-1} \bar{X}_{r}^{q}\right)^{q_{1} / q}\right)^{1 / q_{1}} \\
& \lesssim\left(\sum_{r=1}^{\infty} r^{-q(\varepsilon+(1 / p))-1} \bar{X}_{r}^{q}\left(\sum_{k=1}^{r} k^{\varepsilon q_{1}-1}\right)^{q / q_{1}}\right)^{1 / q} \\
& \lesssim\left(\sum_{r=1}^{\infty} r^{-(q / p)-1} \bar{X}_{r}^{q}\right)^{1 / q}=\|X\|_{N_{p, q}(F)} .
\end{aligned}
$$

To prove the second statement it is enough to show that $\|X\|_{N_{p_{1}, q_{1}}(F)} \leqslant c\|X\|_{N_{p, \infty}(F)}$ and apply the first statement. Since $p_{1}<p$, we have the following:

$$
\begin{aligned}
\|X\|_{N_{p_{1}, q_{1}}(F)} & =\left(\sum_{k=1}^{\infty} k^{-\left(q_{1} / p_{1}\right)-1} \bar{X}_{k}^{q_{1}}\right)^{1 / q_{1}} \\
& \leqslant \sup _{k} k^{-1 / p} \bar{X}_{k}\left(\sum_{k=1}^{\infty} k^{\left(q_{1} / p\right)-\left(q_{1} / p_{1}\right)-1}\right)^{1 / q_{1}} \\
& =c\|X\|_{N_{p, \infty}(F)} .
\end{aligned}
$$

Remark 5. Properties of the $N_{p, q}(F)$ spaces given in Lemma 4 show that the second parameter $q$ is weak with respect to the first $p$. These properties of the spaces are important in the interpolation.

Lemma 6. Let $0<p<\infty, a>1$. If $X \in W(F)$, then for $0<q<\infty$

$$
\|X\|_{N_{p, q}(F)}=\left(\sum_{k=0}^{\infty}\left(a^{-k / p} \bar{X}_{a^{k}}\right)^{q}\right)^{1 / q},
$$

and for $q=\infty$

$$
\|X\|_{N_{p, \infty}(F)}=\sup _{k \in \mathbb{N}} a^{-k / p} \bar{X}_{a^{k}} .
$$

Proof. Using the generalized monotonicity of a process $X$, we have the following:

$$
\begin{aligned}
\|X\|_{N_{p, q}(F)} & =\left(\sum_{k=1}^{\infty} k^{-(q / p)-1} \bar{X}_{k}^{q}\right)^{1 / q} \\
& =\left(\sum_{k=0}^{\infty} \sum_{l=a^{k}}^{a^{k+1}-1} l^{-(q / p)-1} \bar{X}_{l}^{q}\right)^{1 / q}
\end{aligned}
$$




$$
\begin{aligned}
& \gtrsim\left(\sum_{k=0}^{\infty} a^{-k q / p} \bar{X}_{a^{k}}^{q} \sum_{l=a^{k}}^{a^{k+1}-1} \frac{1}{l}\right)^{1 / q} \\
& \gtrsim\left(\sum_{k=0}^{\infty}\left(a^{-k / p} \bar{X}_{a^{k}}\right)^{q}\right)^{1 / q}
\end{aligned}
$$

One can prove the reverse estimate in a similar way.

Lemma 7 (Hölder inequality). Let $0<p_{1}, p_{2}, q<\infty, 0<$ $t, s_{1}, s_{2} \leqslant \infty$ and $(1 / q)=\left(1 / p_{1}\right)+\left(1 / p_{2}\right),(1 / t)=\left(1 / s_{1}\right)+$ $\left(1 / s_{2}\right)$. If stochastic processes $X=\left(X_{n}, \mathbb{G}_{n}\right) \in N_{p_{1}, s_{1}}(F)$ and $Y=\left(Y_{n}, \mathfrak{G}_{n}\right) \in N_{p_{2}, s_{2}}(G)$, then $X Y=\left(X_{n} Y_{n}, \mathfrak{G}_{n}\right) \in N_{q, t}(F)$ and

$$
\|X Y\|_{N_{q, t}(F)} \leqslant\|X\|_{N_{p_{1}, s_{1}}(F)}\|Y\|_{N_{p_{2}, s_{2}}(G)} .
$$

Proof. Since $Y_{n}$ is measurable with respect to an algebra $\mathfrak{G}_{n}$, we have $\overline{X_{k} Y_{k}}(F) \leqslant \overline{X_{k}}(F) \quad \overline{Y_{k}}(G)$ and hence

$$
\begin{aligned}
\|X Y\|_{N_{q, t}(F)}= & \left(\sum_{k=1}^{\infty}\left(k^{-1 / p} \overline{X_{k} Y_{k}}\right)^{t} \frac{1}{k}\right)^{1 / t} \\
\leqslant & \left(\sum_{k=1}^{\infty}\left(k^{-1 / p_{1}} \overline{X_{k}}(F)\right)^{s_{1}} \frac{1}{k}\right)^{1 / s_{1}} \\
& \times\left(\sum_{k=1}^{\infty}\left(k^{-1 / p_{2}} \overline{Y_{k}}(G)\right)^{s_{2}} \frac{1}{k}\right)^{1 / s_{2}} \\
= & \|X\|_{N_{p_{1}, s_{1}}(F)}\|Y\|_{N_{p_{2}, s_{2}}(G)} .
\end{aligned}
$$

We will need the following Hardy-type inequalities.

Lemma 8. Let $s \geqslant 1, \nu>0, \alpha>0, \beta>0$, and $\gamma>0$; then for a nonnegative sequence $a=\left\{a_{k}\right\}_{k}$ the following inequalities hold:

$$
\begin{aligned}
& \left(\sum_{k=1}^{\infty} k^{-\alpha s-1}\left(\sum_{l=1}^{(\gamma k)} l^{\beta-1} a_{l}\right)^{s}\right)^{1 / s} \leqslant C_{\alpha, \beta, s, \gamma}\left(\sum_{k=1}^{\infty} k^{(\beta-\alpha) s-1} a_{k}^{s}\right)^{1 / s}, \\
& \left(\sum_{k=1}^{\infty} k^{\alpha s-1}\left(\sum_{l=1}^{\infty} l^{\beta-1} a_{l}\right)^{s}\right)^{1 / s} \leqslant C_{\alpha, \beta, s, \gamma}\left(\sum_{k=1}^{\infty} k^{(\beta+\alpha) s-1} a_{k}^{s}\right)^{1 / s}, \\
& \left(\sum_{k=0}^{\infty}\left(2^{-\alpha k} \sum_{m=0}^{\gamma k} 2^{\beta m} a_{m}\right)^{s}\right)^{1 / s} \leqslant C_{\alpha, \beta, s, \gamma}\left(\sum_{k=1}^{\infty}\left(2^{(\beta-\alpha) k} a_{k}\right)^{s}\right)^{1 / s}, \\
& \left(\sum_{k=0}^{\infty}\left(2^{\alpha k} \sum_{m=\gamma k}^{\infty} 2^{\beta m} a_{m}\right)^{1 / s} \leqslant C_{\alpha, \beta, s, \gamma}\left(\sum_{k=1}^{\infty}\left(2^{(\beta+\alpha) k} a_{k}\right)^{s}\right)^{1 / s} .\right.
\end{aligned}
$$

\section{Interpolation Method for Stochastic Processes}

Let $\mathbf{T}=\left\{T_{n}\right\}_{n=1}^{\infty}$ be a transform that transforms a stochastic process $X$, which is defined on the system $F=\{\mathfrak{F}\}_{n=1}^{\infty}$, to the stochastic process $\mathbf{T}(X)=\left\{T_{n}(X), \Phi_{n}\right\}_{n=1}^{\infty}$, which is defined on the system $R=\{\mathfrak{R}\}_{n=1}^{\infty}$. We say that the transform $T$ is quasilinear if there exists a constant $C>0$ such that for any $n \in \mathbb{N}$ the following inequality holds almost surely:

$$
\left|T_{n}(X)-T_{n}(Y)\right| \leqslant C\left|T_{n}(X-Y)\right| .
$$

It is known ([9]) that if a process $X=\left(X_{n}, \mathfrak{G}_{n}\right)_{n \geqslant 1}$ is a martingale (submartingale), then the process $X^{\tau}=$ $\left(X_{n \wedge \tau}, \mathfrak{G}_{n}\right)_{n \geqslant 1}$ is also a martingale (submartingale).

Denote $X_{n}^{*}(\omega)=\max _{1 \leqslant k \leqslant n}\left|X_{k}(\omega)\right|$ and $X^{*}=$ $\left(X_{n}^{*}, \mathfrak{G}_{n}\right)_{n \geqslant 1}$.

The transforms $X^{\tau}$ and $X^{*}$ of the stochastic process $X$ are examples of quasilinear transforms.

Let $\mathbf{A}=\left(A_{0}(F), A_{1}(F)\right)$ be a pair of quasinormed own subspaces of linear Hausdorff stochastic processes spaces $\mathfrak{N}(F)$, which is defined on a probability space $(\Omega, \mathfrak{F}, P)$ with a filtration $F=\left\{\mathfrak{F}_{n}\right\}_{n \geqslant 1}$. Obviously, this pair is compatible pair and hence the scale of interpolation spaces is defined with respect to the real method ([12]).

Moreover, let for $0<\theta<1,0<q<\infty$

$$
\begin{aligned}
\left(A_{0}, A_{1}\right)_{\theta, q}=\{X & \in \mathfrak{N}(F):\|X\|_{\left(A_{0}, A_{1}\right)_{\theta, q}}^{q} \\
= & \left.\int_{0}^{\infty}\left(t^{-\theta} K(t, X)\right)^{q} \frac{d t}{t}<\infty\right\}
\end{aligned}
$$

and for $q=\infty$

$$
\begin{aligned}
\left(A_{0}, A_{1}\right)_{\theta, \infty}=\{X & \in \mathfrak{N}(F):\|X\|_{\left(A_{0}, A_{1}\right)_{\theta, \infty}} \\
& \left.=\sup _{0<t<\infty} t^{-\theta} K(t, X)<\infty\right\},
\end{aligned}
$$

where

$$
K\left(t, X ; A_{0}, A_{1}\right)=\inf _{X=X_{0}+X_{1}}\left(\left\|X_{0}\right\|_{A_{0}}+t\left\|X_{1}\right\|_{A_{1}}\right)
$$

is the Peetre functional.

Let $R=\left\{\tau_{k}(\omega)\right\}_{k=1}^{\infty}$ be a sequence of stopping times with respect to a filtration $F$ and $A(F)=\left(A_{0}(F), A_{1}(F)\right)$ be a pair of quasinormed own subspaces $\mathfrak{N}(F)$. Let $X \in \mathfrak{N}(F)$ and $t \epsilon$ $(0, \infty)$. We define the following:

$$
\begin{aligned}
K_{R}(t, X) & =K\left(t, X ; A_{0}, A_{1}, R\right) \\
& =\inf _{\tau \in R \cup\{0\}}\left(\left\|X-X^{\tau}\right\|_{A_{0}}+t\left\|X^{\tau}\right\|_{A_{1}}\right) .
\end{aligned}
$$

Here the infimum is taken over all stopping times from $R$. Moreover for $0<q<\infty$

$$
\begin{aligned}
\left(A_{0}, A_{1}\right)_{\theta, q}^{R}=\{X & \in \mathfrak{N}(F):\|X\|_{\left(A_{0}, A_{1}\right)_{\theta, q}}^{q} \\
= & \left.\int_{0}^{\infty}\left(t^{-\theta} K_{R}(t, X)\right)^{q} \frac{d t}{t}<\infty\right\},
\end{aligned}
$$


and for $q=\infty$

$$
\begin{aligned}
\left(A_{0}, A_{1}\right)_{\theta, \infty}^{R}=\{X & \in \mathfrak{N}(F):\|X\|_{\left(A_{0}, A_{1}\right)_{\theta, \infty}} \\
= & \left.\sup _{0<t<\infty} t^{-\theta} K_{R}(t, X)<\infty\right\} .
\end{aligned}
$$

Theorem 9. Let $\left(A_{0}(F), A_{1}(F)\right),\left(B_{0}(\Phi), B_{1}(\Phi)\right)$ be two compatible pairs of stochastic processes and let $R=\{\tau(\omega)\}$ be some fixed family of Markov times with respect to a filtration $F$. If T is a quasilinear map for stochastic processes $X=\left(X_{n}, \mathfrak{F}_{n}\right)_{n \geqslant 1}$ and

$$
\begin{gathered}
\left\|T\left(X-X^{\tau}\right)\right\|_{B_{0}} \leqslant M_{0}\left\|X-X^{\tau}\right\|_{A_{0}}, \\
\left\|T\left(X^{\tau}\right)\right\|_{B_{1}} \leqslant M_{1}\left\|X^{\tau}\right\|_{A_{1}}
\end{gathered}
$$

for all stopping times $\tau \in R$, then

$$
\|T(X)\|_{\mathbf{B}_{\theta, \mathbf{q}}} \leqslant C M_{0}^{1-\theta} M_{1}^{\theta}\|X\|_{\mathbf{A}_{\theta, q}^{R},}
$$

where the constant $C$ is from the definition of quasilinearity of the operator $T$.

Proof. Consider the following:

$$
\begin{aligned}
& \|T(X)\|_{\mathbf{B}_{\theta, \mathbf{q}}} \\
& =\left(\int_{0}^{\infty}\left(t^{-\theta} \inf _{T(X)=Y_{0}+Y_{1}}\left(\left\|Y_{0}\right\|_{B_{0}}+t\left\|Y_{1}\right\|_{B_{1}}\right)\right)^{q} \frac{d t}{t}\right)^{1 / q} \\
& \leqslant\left(\int _ { 0 } ^ { \infty } \left(t ^ { - \theta } \operatorname { i n f } _ { \tau \in R \cup \{ 0 \} } \left(\left\|T(X)-T\left(X^{\tau}\right)\right\|_{B_{0}}\right.\right.\right. \\
& \left.\left.\left.+t\left\|T\left(X^{\tau}\right)\right\|_{B_{1}}\right)\right)^{q} \frac{d t}{t}\right)^{1 / q} \\
& \leqslant C\left(\int _ { 0 } ^ { \infty } \left(t ^ { - \theta } \operatorname { i n f } _ { \tau \in R \cup \{ 0 \} } \left(\left\|T\left(X-X^{\tau}\right)\right\|_{B_{0}}\right.\right.\right. \\
& \left.\left.\left.+t\left\|T\left(X^{\tau}\right)\right\|_{B_{1}}\right)\right)^{q} \frac{d t}{t}\right)^{1 / q} \\
& \leqslant C M_{0}\left(\int _ { 0 } ^ { \infty } \left(t ^ { - \theta } \operatorname { i n f } _ { \tau \in R \cup \{ 0 \} } \left(\left\|X-X^{\tau}\right\|_{A_{0}}\right.\right.\right. \\
& \left.\left.\left.+t \frac{M_{1}}{M_{0}}\left\|X^{\tau}\right\|_{A_{1}}\right)\right)^{q} \frac{d t}{t}\right)^{1 / q} \\
& =C M_{0}^{1-\theta} M_{1}^{\theta}\|X\|_{\mathbf{A}_{\theta, q}^{R}(F)} \text {. }
\end{aligned}
$$

The theorem is proved.
Lemma 10. Let $a>1$ and $R=\{k\}_{k \in \mathbb{N}}$ be stopping times. Then for $0<q<\infty$

$$
\begin{aligned}
\|X\|_{\left(A_{0}, A_{1}\right)_{\theta, q}^{R}} & =\left(\sum_{n=-\infty}^{\infty}\left(a^{-\theta n} K_{R}\left(a^{n}, X\right)\right)^{q}\right)^{1 / q}, \\
\|X\|_{\left(A_{0}, A_{1}\right)_{\theta, q}} & =\left(\sum_{n=-\infty}^{\infty}\left(a^{-\theta n} K\left(a^{n}, X\right)\right)^{q}\right)^{1 / q}, \\
\|X\|_{\left(A_{0}, A_{1}\right)_{\theta, \infty}^{R}} & =\sup _{n \in \mathbb{Z}} a^{-\theta n} K_{R}\left(a^{n}, X\right), \\
\|X\|_{\left(A_{0}, A_{1}\right)_{\theta, \infty}} & =\sup _{n \in \mathbb{Z}} a^{-\theta n} K\left(a^{n}, X\right) .
\end{aligned}
$$

The proof is similar to the proof of the Lemma 4.

\section{Interpolation Properties of the Spaces $N_{p, q}(F)$}

Theorem 11. Let $1<p_{0}<p_{1}<\infty, 1 \leqslant q_{0}, q_{1}, q \leqslant \infty, 0<$ $\theta<1,(1 / p)=\left((1-\theta) / p_{0}\right)+\left(\theta / p_{1}\right)$, and $R=\{k\}_{k \in \mathbb{N}}$ be the stopping times. Then for any stochastic process $X=$ $\left(X_{n}, \mathfrak{F}_{n}\right)_{n \geqslant 1}$,

$$
\|X\|_{N_{p, q}(F)} \leqslant c\|X\|_{\left(N_{p_{0}, q_{0}}(F), N_{p_{1}, q_{1}}(F)\right)_{\theta, q}},
$$

where the constant $c$ depends only on parameters $p_{i}, q_{i}, \theta, i=$ 0,1 .

If $X=\left(X_{n}, \mathfrak{\mho}_{n}\right)_{n \geqslant 1} \in W(F)$, then

$$
\|X\|_{\left(N_{p_{0}, q_{0}}(F), N_{p_{1}, q_{1}}(F)\right)_{\theta, q}^{R}} \leqslant c\|X\|_{N_{p, q}(F)},
$$

where the constant $c$ also depends only on parametres $p_{i}, q_{i}, \theta$, $i=0,1$.

Proof. Let $X=Y+Z$ be any representation of a process $X$, where $Y \in N_{p_{0}, q_{0}}(F), Z \in N_{p_{1}, q_{1}}(F)$. To prove the first statement of the theorem, we use the following inequality: $\bar{X}_{m} \leqslant \bar{Y}_{m}+\bar{Z}_{m}$.

For any $a>1$ we have the following:

$$
\begin{aligned}
a^{-n / p} & \bar{X}_{a^{n}} \\
\leqslant & a^{-(n / p)+\left(n / p_{0}\right)}\left(a^{-n / p_{0}} \bar{Y}_{a^{n}}+a^{-\left(n / p_{0}\right)+\left(n / p_{1}\right)} a^{-n / p_{1}} \bar{Z}_{a^{n}}\right) \\
\leqslant & a^{-(n / p)+\left(n / p_{0}\right)} \\
& \times\left(\sup _{n} a^{-n / p_{0}} \bar{Y}_{a^{n}}+a^{-\left(n / p_{0}\right)+\left(n / p_{1}\right)} \sup _{n} a^{-n / p_{1}} \bar{Z}_{a^{n}}\right) \\
= & a^{\theta\left(\left(1 / p_{0}\right)-\left(1 / p_{1}\right)\right) n} \\
& \times\left(\|Y\|_{N_{p_{0}, \infty}(F)}+a^{-\left(\left(1 / p_{0}\right)-\left(1 / p_{1}\right)\right) n}\|Z\|_{N_{p_{1}, \infty}(F)}\right) .
\end{aligned}
$$


By putting $a=2^{p_{0} p_{1} /\left(p_{1}-p_{0}\right)}$, we get $a^{-n / p} \bar{X}_{n} \leqslant 2^{n \theta} K\left(2^{-n}, X\right)$. Therefore, using (22) and Lemma 8, we have the following:

$$
\begin{aligned}
\|X\|_{N_{p, q}(F)} & =\left(\sum_{n=-\infty}^{\infty}\left(a^{-n / p} \bar{X}_{n}\right)^{q}\right)^{1 / q} \\
& \leqslant\left(\sum_{n=-\infty}^{\infty}\left(2^{n \theta} K\left(2^{-n}, X\right)\right)^{q}\right)^{1 / q} \\
& =\left(\sum_{n=-\infty}^{\infty}\left(2^{-n \theta} K\left(2^{n}, X\right)\right)^{q}\right)^{1 / q} \\
& =\|X\|_{\left(N_{p_{0}, \infty}(F), N_{p_{1}, \infty}(F)_{\theta_{\theta, q}}\right.}
\end{aligned}
$$

Let us prove the second statement of the theorem. Let $X=$ $\left(X_{n}, \mathfrak{\mho}_{n}\right)_{n \geqslant 1} \in W(F)$. By using Lemmas 8 and 3 we have the following:

$$
\begin{aligned}
& \|X\|_{\left(N_{p_{0}, q_{0}}(F), N_{p_{1}, q_{1}}(F)\right)_{\theta, q}^{R}} \\
& =\left(\sum_{n=-\infty}^{\infty}\left(2^{\theta n} K_{R}\left(2^{-n}, X\right)\right)^{q}\right)^{1 / q} \\
& =\left(\sum _ { n = - \infty } ^ { \infty } \left(2 ^ { \theta n } \operatorname { i n f } _ { k \geqslant 0 } \left(\left\|X-X^{k}\right\|_{N_{p_{0}, q_{0}}}\right.\right.\right. \\
& \left.\left.\left.+2^{-n}\left\|X^{k}\right\|_{N_{p_{1}, q_{1}}}\right)\right)^{q}\right)^{1 / q} \\
& \leqslant\left(\sum _ { n = - \infty } ^ { \infty } \left(2 ^ { \theta n } \operatorname { i n f } _ { k \geqslant 0 } \left(\left\|X-X^{k}\right\|_{N_{p_{0}, 1}}\right.\right.\right. \\
& \left.\left.\left.+2^{-n}\left\|X^{k}\right\|_{N_{p_{1}, 1}}\right)\right)^{q}\right)^{1 / q} .
\end{aligned}
$$

Let $k=\left[2^{n \gamma}\right]$, then taking into account that $X^{2^{n \gamma}} \equiv 0, n \leqslant-1$, we obtain the following:

$$
\begin{aligned}
& \|X\|_{\left(N_{p_{0}, q_{0}}(F), N_{p_{1}, q_{1}}(F)\right)_{\theta, q}^{R}} \\
& \lesssim\left(\sum_{n=-\infty}^{\infty}\left(2^{\theta n}\left(\left\|X-X^{2^{n \gamma}}\right\|_{N_{p_{0}, 1}}+2^{-n}\left\|X^{2^{n \gamma}}\right\|_{N_{p_{1}, 1}}\right)\right)^{q}\right)^{1 / q} \\
& =\left(\sum_{n=0}^{+\infty}\left(2^{\theta n}\left(\left\|X-X^{2^{n \gamma}}\right\|_{N_{p_{0}, 1}}+2^{-n}\left\|X^{2^{n \gamma}}\right\|_{N_{p_{1}, 1}}\right)\right)^{q}\right)^{1 / q} \\
& +\left(\sum_{n=-\infty}^{-1}\left(2^{\theta n}\left(\|X\|_{N_{p_{0}, 1}}\right)\right)^{q}\right)^{1 / q} \\
& =\left(\sum_{n=0}^{\infty}\left(2^{\theta n}\left(\left\|X-X^{2^{n \gamma}}\right\|_{N_{p_{0}, 1}}+2^{-n}\left\|X^{2^{n \gamma}}\right\|_{N_{p_{1}, 1}}\right)\right)^{q}\right. \\
& \left.\quad+\|X\|_{N_{p_{0}, 1}}^{q} \sum_{n=1}^{+\infty} 2^{-\theta n q}\right)^{1 / q} .
\end{aligned}
$$

Thus, we obtain the following:

$$
\begin{aligned}
& \|X\|_{\left(N_{p_{0}, q_{0}}(F), N_{p_{1}, q_{1}}(F)\right)_{\theta, q}^{R}} \\
& \leq\left(\sum_{n=0}^{\infty}\left(2^{\theta n}\left(\left\|X-X^{2^{n \gamma}}\right\|_{N_{p_{0}, 1}}+2^{-n}\left\|X^{2^{n \gamma}}\right\|_{N_{p_{1}, 1}}\right)\right)^{q}\right. \\
& \left.\quad+\|X\|_{N_{p_{0}, 1}^{q}}^{q}\right)^{1 / q} .
\end{aligned}
$$

Further, we have the following:

$$
\begin{aligned}
\| X & -X^{2^{n \gamma}} \|_{N_{p_{0}, 1}} \\
& =\sum_{k=1}^{\infty} k^{-1 / p_{0}} \overline{\left(X-X^{2^{n \gamma}}\right)_{k}}=\sum_{k=0}^{\infty} 2^{-k / p_{0}} \overline{\left(X-X^{2^{n \gamma}}\right)_{2^{k}}} \\
& =\sum_{k=n \gamma}^{\infty} 2^{-k / p_{0}} \overline{\left(X-X^{2^{n \gamma}}\right)_{2^{k}}} \leqslant(1+C) \sum_{k=n \gamma}^{\infty} 2^{-k / p_{0}} \bar{X}_{2^{k}},
\end{aligned}
$$

$$
\begin{aligned}
\| X^{2^{n \gamma}} & \|_{p_{1}, 1} \\
= & \sum_{k=1}^{\infty} k^{-1\left(1 / p_{1}\right)} \overline{X_{k}^{2^{n \gamma}}}=\sum_{k=0}^{\infty} 2^{-k / p_{1}} \overline{X_{2^{k}}^{2^{n \gamma}}} \\
& \leqslant \sum_{k=0}^{n \gamma} 2^{-k / p_{1}} \bar{X}_{2^{k}}+\bar{X}_{2^{n \gamma}} \sum_{k=n \gamma}^{\infty} 2^{-k / p_{1}} \\
& \leqslant C\left(\sum_{k=0}^{n \gamma} 2^{-k / p_{1}} \bar{X}_{2^{k}}+2^{n \gamma\left(\left(1 / p_{0}\right)-\left(1 / p_{1}\right)\right)} \sum_{k=n \gamma}^{\infty} 2^{-k / p_{0}} \bar{X}_{2^{k}}\right) .
\end{aligned}
$$

By using Lemma 8 for $(1 / \gamma)=\left(1 / p_{0}\right)-\left(1 / p_{1}\right),(46)$, and $(47)$, we have the following:

$$
\begin{gathered}
\left(\sum_{n=0}^{\infty}\left(2^{\theta n}\left\|X-X^{2^{n \gamma}}\right\|_{N_{p_{0}, 1}}\right)^{q}\right)^{1 / q} \\
\leq\left(\sum_{n=0}^{\infty}\left(2^{\theta n} \sum_{k=n \gamma}^{\infty} 2^{-k / p_{0}} \bar{X}_{2^{k}}\right)^{q}\right)^{1 / q} \\
\leq\left(\sum_{k=0}^{\infty}\left(2^{-k / p} \bar{X}_{2^{k}}\right)^{q}\right)^{1 / q}=\|X\|_{N_{p, q(F)}},
\end{gathered}
$$




$$
\begin{aligned}
& \left(\sum_{n=0}^{\infty} 2^{-(1-\theta) n q}\left\|X^{2^{n \gamma}}\right\|_{N_{p_{1}, 1}}^{q}\right)^{1 / q} \\
& \leq\left(\sum _ { n = 0 } ^ { \infty } 2 ^ { - n q ( 1 - \theta ) } \left(\sum_{k=0}^{n \gamma} 2^{-k / p_{1}} \bar{X}_{2^{k}}\right.\right. \\
& \left.\left.+2^{n \gamma\left(\left(1 / p_{0}\right)-\left(1 / p_{1}\right)\right)} \sum_{k=n \gamma}^{\infty} 2^{-k / p_{0}} \bar{X}_{2^{k}}\right)^{q}\right)^{1 / q} \\
& \leq\left(\sum_{k=0}^{\infty}\left(2^{-k / p} \bar{X}_{2^{k}}\right)^{q}\right)^{1 / q}=\|X\|_{N_{p, q}}(F) .
\end{aligned}
$$

By applying Minkowski's inequality to (45) and using Lemma 4, estimates (48), we obtain

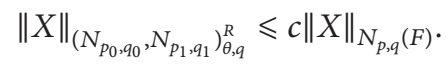

Corollary 12. Let $0<p_{0}<p_{1}<\infty, 0<q_{0}<q_{1}<\infty, 0<$ $\theta<1,1 \leqslant s \leqslant \infty,(1 / q)=\left((1-\theta) / q_{0}\right)+\left(\theta / q_{1}\right),(1 / p)=((1-$ $\left.\theta) / p_{0}\right)+\left(\theta / p_{1}\right), X \in W(F)$, and $T=\left\{T_{n}\right\}_{n=1}^{\infty}$ be a quasilinear transform. If for any $k \in \mathbb{N} \cup\{0\}$ the following conditions hold:

$$
\begin{gathered}
\left\|T\left(X^{k}\right)\right\|_{N_{q_{1}, \infty}(R)} \leqslant M_{1}\left\|X^{k}\right\|_{N_{p_{1}, 1}(F)}, \\
\left\|T\left(X-X^{k}\right)\right\|_{N_{q_{0}, \infty}(R)} \leqslant M_{0}\left\|X-X^{k}\right\|_{N_{p_{0}, 1}(F)},
\end{gathered}
$$

then

$$
\|T(X)\|_{N_{q, s}(R)} \leqslant C M_{0}^{1-\theta} M_{1}^{\theta}\|X\|_{N_{p, s}(F)},
$$

where $C>0$ depends only on $p_{0}, p_{1}, q_{0}, q_{1}$, and $\theta$.

Taking into account that the measurable function may be considered as a martingale, by corollary we may receive Marcinkiewicz-Calderon interpolation theorem (see [13]).

Corollary 13 (Marcinkiewicz-Calderon theorem). Let $0<$ $p_{0}<p_{1}<\infty, 0<q_{0}<q_{1} \leqslant \infty, \theta \in(0,1), 1 / p=$ $(1-\theta) / p_{0}+\theta / p_{1}$, and $1 / q=(1-\theta) / q_{0}+\theta / q_{1}$. If $T$ is a quasilinear map and

$$
\begin{gathered}
T: L_{p_{i}, 1}(D, v) \longrightarrow L_{q_{i}, \infty}(\Omega, \mu) \\
\text { with the norm } M_{i}, i=0,1,
\end{gathered}
$$

then

$$
T: L_{p, \tau}(D, \nu) \longrightarrow L_{q, \tau}(\Omega, \mu), \quad\|T\| \leqslant M_{0}^{1-\theta} M_{1}^{\theta} .
$$

Corollary 14. Let $T=\left\{T_{n}\right\}_{n=1}^{\infty}$ be a quasilinear transform such that for any $p \in(a, b)$ and for any $X \in N_{p, 1}(F) \cap W(F)$ the following weak inequality holds:

$$
\|T(X)\|_{N_{p, \infty}(R)} \leqslant C_{p}\|X\|_{N_{p, 1}(F)} .
$$

Then

$$
\|T(X)\|_{N_{p, s}(R)} \leqslant C_{p, s}\|X\|_{N_{p, s}(F)}, \quad X \in N_{p, s}(F) \cap W(F)
$$

for any $p$ and $s$ such that $p \in(a, b), 1 \leqslant s \leqslant \infty$.

\section{Boundedness of Some Operators in Class $N_{p, q}(F)$}

Let $Y=\left(Y_{n}, \mathfrak{G}_{n}\right)_{n \geqslant 0}$ be a stochastic sequence and $V=$ $\left(V_{n}, \mathfrak{G}_{n-1}\right)$ be a predicted sequence $\left(\mathfrak{G}_{-1}=\mathfrak{G}_{0}\right)$. A stochastic sequence $V \cdot Y=\left((V \cdot Y)_{n}, \mathfrak{G}_{n}\right)$ such that

$$
(V \cdot Y)_{n}=V_{0} Y_{0}+\sum_{i=1}^{n} V_{i} \Delta Y_{i},
$$

where $\Delta Y_{i}=Y_{i}-Y_{i-1}$ is called the transform of $Y$ with respect to $V$. If $Y$ is a martingale then we say that $V \cdot Y$ is the martingale transform.

Theorem 15. Let $0<q<p<\infty, 1 \leqslant \tau \leqslant \infty$ and $1 / r=$ $1 / q-1 / p$. Let $V \cdot Y$ be a martingale transform of a martingale $Y$ by predicted sequence $V=\left(V_{n}, \mathfrak{F}_{n-1}\right)$. If

$$
\|V\|_{N_{r, \infty}(G)}+\left\|\left(n \Delta V_{n}\right)\right\|_{N_{r, \infty}(G)} \leqslant B,
$$

then

$$
\|V \cdot Y\|_{N_{q, \tau}(G)} \leqslant c B\|Y\|_{N_{p, \tau}(G)},
$$

where a constant $c$ depends only on parametres $p, q$, and $\tau$.

Proof. Let $V \cdot Y$ be a martingale transform of a martingale $Y$ by predicted sequence $V=\left(V_{n}, \mathfrak{F}_{n-1}\right)$; that is,

$$
(V \cdot Y)_{n}=\sum_{k=1}^{n} V_{k} \Delta Y_{k},
$$

where $Y_{0}=0, \Delta Y_{k}=Y_{k}-Y_{k-1}$. By Abel's transform $(V \cdot Y)_{n}=$ $\sum_{k=1}^{n-1} \Delta V_{k} Y_{k}+V_{n} Y_{n}$, we get the following:

$$
\begin{aligned}
\|V \cdot Y\|_{N_{q, \infty}(G)} & =\sup _{n \in N} n^{-1 / q} \overline{(V \cdot Y)_{n}} \\
& \leqslant \sup _{n \in N} n^{-1 / q}\left(\sum_{k=1}^{n} \overline{\Delta V_{k} Y_{k}}+\overline{V_{n} Y_{n}}\right) .
\end{aligned}
$$

Taking into account that $\Delta V_{k}, Y_{k}$ are measurable functions with respect to the algebra $\mathfrak{G}_{k}$, we have $\overline{\Delta V_{k} Y_{k}} \leqslant$ $\overline{\Delta V_{k}} \overline{Y_{k}}, \overline{V_{n} Y_{n}} \leqslant \overline{V_{n} Y_{n}}$ and

$$
\begin{aligned}
\sup _{n \in N} n^{-1 / q}(\overline{V \cdot Y})_{n} \leqslant & \sup _{n \in N} \sum_{k=1}^{n}\left(k^{-1 / q} k \overline{\Delta V_{k}}\right)\left(k^{-(1 / p)-1} \overline{Y_{k}}\right) \\
& +n^{-1 / r} \overline{V_{n}} n^{-1 / p} \overline{Y_{n}} \\
\leqslant & \sup _{k} k^{1-(1 / r)} \overline{\Delta V_{k}} \cdot\|Y\|_{N_{p, 1}(G)} \\
& +\left\|V_{n}\right\|_{N_{r, \infty}(G)}\|Y\|_{N_{p, \infty}(G)} \\
\leqslant & B\|Y\|_{N_{p, 1}(G)} .
\end{aligned}
$$


Hence the weak inequality is proved as follows:

$$
\|V \cdot Y\|_{N_{q, \infty}(G)} \leqslant B\|Y\|_{N_{p, 1}(G)}
$$

for $1<q<p<\infty$

Let $0<q<p<\infty,(1 / r)=(1 / q)-(1 / p), 0<q_{0}<q<$ $q_{1}<\infty$ and $0<p_{0}<p<p_{1}<\infty$. Let a pair of numbers $\left(p_{0}, p_{1}\right)$ and $\left(q_{0}, q_{1}\right)$ satisfy the following condition:

$$
\frac{1}{q_{0}}-\frac{1}{p_{0}}=\frac{1}{q_{1}}-\frac{1}{p_{1}}=\frac{1}{q}-\frac{1}{p} .
$$

Then from that is proved above it follows that

$$
\begin{gathered}
\|V \cdot Y\|_{N_{q_{0}, \infty}(G)} \leqslant B\|Y\|_{N_{p_{0}, 1}(G)}, \\
\|V \cdot Y\|_{N_{q_{1}, \infty}(G)} \leqslant B\|Y\|_{N_{p_{1}, 1}(G)}
\end{gathered}
$$

for $0<p<q<\infty$.

Taking into account that for any stopping time $k \in N$ processes $Y^{k}$ and $Y-Y^{k}$ are martingales, it is possible to apply Theorem 9. Then

$$
\|V \cdot Y\|_{N_{q_{\theta}, \tau}} \leqslant c B\|Y\|_{N_{p_{\theta}, \tau}}
$$

where

$$
\frac{1}{q_{\theta}}=\frac{1-\theta}{q_{0}}+\frac{\theta}{q_{1}}, \quad \frac{1}{p_{\theta}}=\frac{1-\theta}{p_{0}}+\frac{\theta}{p_{1}} .
$$

Note that there exists $\theta \in(0,1)$ such that $\left(1 / p_{\theta}\right)=(1 / p)$. Then it follows from $(63)$ that $\left(1 / q_{\theta}\right)=(1 / q)$.

Theorem 16. Let $0<p<\infty, 1 \leqslant q \leqslant \infty$ and $X=$ $\left(X_{n}, \mathfrak{G}_{n}\right)_{n \geqslant 1} \in W(G)$ and $\tau(\omega)$ be the Markov time and let $X^{\tau}=\left(X_{n \wedge \tau}, \mathfrak{G}_{n}\right)_{n \geqslant 1}$ be a stopped process. Then

$$
\left\|X^{\tau}\right\|_{N_{p, q}(G)} \leqslant c\|X\|_{N_{p, q}(G)} .
$$

Proof. Denote

$$
\begin{gathered}
W_{r}=\{\omega: \tau(\omega)=r\} \in \mathfrak{G}_{r}, \quad r=\overline{1, n-1}, \\
W_{n}=\{\omega: \tau(\omega) \geqslant n\} \in \mathfrak{G}_{n} .
\end{gathered}
$$

Let us show that $\left\|X^{\tau}\right\|_{N_{p, \infty}(G)} \leqslant c\|X\|_{N_{p, 1}(G)}$.

$$
\text { If } 2^{s} \leqslant n<2^{s+1} \text {, then }
$$

$$
\begin{aligned}
& n^{-1 / p} \sup _{A \in \mathfrak{S}_{n}, P(A)>0} \frac{1}{P(A)}\left|\int_{A} X^{\tau} P(d \omega)\right| \\
& =n^{-1 / p} \sup _{A \in \mathfrak{G}_{n}, P(A)>0} \frac{1}{P(A)}\left|\sum_{r=1}^{n} \int_{A \cap W_{r}} X_{r} P(d \omega)\right| \\
& \leqslant 2^{-s / p} \sup _{A \in \mathfrak{G}_{2^{s+1}-1}} \frac{1}{P(A)} \sum_{t=0}^{s} \sum_{r=2^{t}}^{2^{t+1}-1}\left|\int_{A \cap W_{r}} X_{r} P(d \omega)\right| \\
& \leqslant 2^{-s / p} \sup _{A \in \mathfrak{G}_{2^{s+1}-1}} \frac{1}{P(A)} \sum_{t=0}^{s} \sum_{r=2^{t}}^{2^{t+1}-1} P\left(A \cap W_{r}\right) \overline{X_{r}} \\
& \leqslant 2^{-s / p} \sup _{A \in \mathfrak{G}_{2^{s+1}-1}} \frac{1}{P(A)} \sum_{t=0}^{s} \bar{X}_{2^{t+1}-1} \sum_{r=2^{t}}^{2^{t+1}-1} P\left(A \cap W_{r}\right) \\
& =2^{-s / p} \sup _{A \in \mathfrak{S}_{2^{s+1}-1}} \sum_{t=0}^{s} \bar{X}_{2^{t+1}-1} \sum_{r=2^{t}}^{2^{t+1}-1} P\left(W_{r} \mid A\right) \\
& \leqslant \sum_{t=0}^{s} 2^{-t / p} \bar{X}_{2^{t+1}-1} \leqslant 2^{1 / p} \sum_{t=0}^{s} 2^{-(t+1) / p} \bar{X}_{2^{t+1}} \\
& =2^{1 / p} \sum_{k=0}^{s+1} 2^{-k / p} \bar{X}_{2^{k}} \leqslant c\|X\|_{N_{p, 1}(G)} .
\end{aligned}
$$

Now, using Corollary 12 we get the statement of the Theorem 16.

Corollary 17. Let $0<p<\infty, 1 \leqslant q \leqslant \infty$ and a process $X=\left(X_{n}, \mathfrak{G}_{n}\right)_{n \geqslant 1}$ be a nonnegative submartingale. Then the process $X^{*}=\left(X_{n}^{*}, \mathfrak{G}_{n}\right)_{n \geqslant 1}$ is also submartingale and

$$
\left\|X^{*}\right\|_{N_{p, q}(G)} \sim\|X\|_{N_{p, q}(G)} .
$$

Proof. It follows from Theorem 16 that

$$
\left\|X^{*}\right\|_{N_{p, q}(G)} \leqslant c\|X\|_{N_{p, q}(G)} .
$$

The reverse inequality is trivial.

\section{Interpolation Properties of the Space $N_{p}^{\alpha, q}(G)$, the Embedding Theorems}

Theorem 18. Let $1 \leqslant p, q, q_{0}, q_{1} \leqslant \infty, \alpha_{0}<\alpha_{1}, 0<\theta<$ $1, \alpha=(1-\theta) \alpha_{0}+\theta \alpha_{1}$, and $R=\{r\}_{r \in \mathbb{N}}$. Then

$$
\left(N_{p}^{\alpha_{0}, q_{0}}(G), N_{p}^{\alpha_{1}, q_{1}}(G)\right)_{\theta, q}^{R}=N_{p}^{\alpha, q}(G)
$$


Proof. Using Lemmas 10 and 4 we have the following:

$$
\begin{aligned}
& \|X\|_{\left(N_{p}^{\alpha_{0}, q_{0}}, N_{p}^{\alpha_{1}, q_{1}, R}\right)_{\theta, q}^{R}} \\
& \asymp\left(\sum_{n=-\infty}^{\infty}\left(2^{\theta n} K_{R}\left(2^{-n}, X\right)\right)^{q}\right)^{1 / q} \\
& =\left(\sum_{n=-\infty}^{\infty}\left(2^{\theta n} \inf _{r \geqslant 0}\left(\left\|X-X^{r}\right\|_{N_{p}^{\alpha_{0}, q_{0}}}+2^{-n}\left\|X^{r}\right\|_{N_{p}^{\alpha_{1}, q_{1}}}\right)\right)^{q}\right)^{1 / q} \\
& \leq\left(\sum_{n=-\infty}^{\infty}\left(2^{\theta n} \inf _{r \geqslant 0}\left(\left\|X-X^{r}\right\|_{N_{p}^{\alpha_{0}, 1}}+2^{-n}\left\|X^{r}\right\|_{N_{p}^{\alpha_{1}, 1}}\right)\right)^{q}\right)^{1 / q} .
\end{aligned}
$$

Putting $r=\left[2^{n \gamma}\right], \gamma=\alpha_{1}-\alpha_{0}$ we get the following:

$$
\begin{aligned}
& \|X\|_{\left(N_{p}^{\alpha_{0}, q_{0}}, N_{p}^{\alpha_{1}, q_{1}, R}\right)_{\theta, q}^{R}} \\
& \leq\left(\sum_{n=0}^{\infty}\left(2^{\theta n}\left(\left\|X-X^{2^{n \gamma}}\right\|_{N_{p}^{\alpha_{0}, 1}}+2^{-n}\left\|X^{2^{n \gamma}}\right\|_{N_{p}^{\alpha_{1}, 1}}\right)\right)^{q}\right. \\
& \left.\quad+\|X\|_{N_{p}^{\alpha_{0}, 1}}^{q}\right)^{1 / q} .
\end{aligned}
$$

It follows from the definition that $\overline{\left(\Delta X^{2^{r}}\right)_{k}}=\overline{\Delta X}_{k}$ for $k=$ $0,1 \ldots, r-1 ;{\overline{\left(\Delta X^{2^{r}}\right)_{k}}}=0$ for $k \geqslant r, \overline{\Delta\left(X-X^{2^{r}}\right)_{k}}=0$ for $k=0,1 \ldots, r-1 ;{\overline{\Delta\left(X-X^{2^{r}}\right)_{k}}}=\overline{\Delta X}_{k}$ for $k \geqslant r$; therefore,

$$
\begin{gathered}
\left\|X^{2^{r}}\right\|_{N_{p}^{\alpha, 1}(G)}=\sum_{k=0}^{r-1} 2^{\alpha k} \overline{\Delta X}_{k}, \\
\left\|X-X^{2^{r}}\right\|_{N_{p}^{\alpha, 1}(G)}=\sum_{k=r}^{\infty} 2^{\alpha k} \overline{\Delta X}_{k} .
\end{gathered}
$$

Substituting these equalities in (74) and applying Lemmas 4 and 8 , we get the following:

$$
\begin{gathered}
\|X\|_{\left(N_{p}^{\alpha_{0} q_{0}}, N_{p}^{\alpha_{1} q_{1}}\right)_{\theta, q}^{R}} \\
\leq\left(\sum_{n=0}^{\infty} 2^{\theta n q}\left(\sum_{k=n \gamma}^{\infty} 2^{\alpha_{0} k} \overline{\Delta X}_{k}+2^{-n} \sum_{k=0}^{n \gamma-1} 2^{\alpha_{1} k} \overline{\Delta X}_{k}\right)^{q}\right. \\
\left.+\|X\|_{N_{p}^{\alpha_{0}, 1}}^{q}\right)^{1 / q} \\
\leq\left(\sum_{n=0}^{\infty}\left(2^{\left(\alpha_{0}+\theta \gamma\right) n} \overline{\Delta X}_{k}\right)^{q}\right)^{1 / q} \\
+\left(\sum_{n=0}^{\infty}\left(2^{\left(\alpha_{1}-(1-\theta) \gamma\right) n} \overline{\Delta X}_{k}\right)^{q}\right)^{1 / q}+\|X\|_{N_{p}^{\alpha, q}} \\
=3\|X\|_{N_{p}^{\alpha, q} .}
\end{gathered}
$$

For the proof of reverse estimate we use the fact that for any $r$ and $k$ the following equality holds:

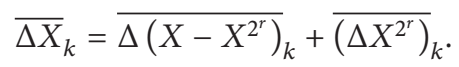

Then we have the following:

$$
\begin{aligned}
& \|X\|_{N_{p}^{\alpha, q}} \\
& =\left(\sum_{k=0}^{\infty}\left(2^{\alpha k} \overline{\Delta X}_{k}\right)^{q}\right)^{1 / q} \\
& \leqslant\left(\sum _ { k = 0 } ^ { \infty } \left(2 ^ { \alpha k - \alpha _ { 0 } k } \left(\sup _{n \geqslant 0} 2^{\alpha_{0} n} \overline{\Delta\left(X-X^{2^{r}}\right)_{n}}\right.\right.\right. \\
& \left.\left.\left.+2^{\alpha_{0} k-\alpha_{1} k} \cdot \sup _{n \geqslant 0} 2^{\alpha_{1} n} \overline{\left(\Delta X^{2^{r}}\right)_{n}}\right)\right)^{q}\right)^{1 / q} \\
& =\left(\sum _ { k = 0 } ^ { \infty } \left(2 ^ { \alpha k - \alpha _ { 0 } k } \left(\left\|X-X^{2^{r}}\right\|_{N_{p}^{\alpha_{0}, \infty}}\right.\right.\right. \\
& \left.+2^{\alpha_{0} k-\alpha_{1} k} \|_{\left.\left.X^{2^{r}} \|_{N_{p}^{\alpha_{1}, \infty}}\right)\right)^{q}}\right)^{\frac{1}{q}} .
\end{aligned}
$$

Substituting $2^{\alpha_{1}-\alpha_{0}}=a$ and using Lemma 4, we have the following:

$$
\begin{aligned}
\|X\|_{N_{p}^{\alpha, q}} & \leqslant\left(\sum_{k=0}^{\infty}\left(a^{\theta k} \cdot K\left(a^{-k}, X, N_{p}^{\alpha_{0}, \infty}, N_{p}^{\alpha_{1}, \infty}\right)\right)^{q}\right)^{1 / q} \\
& \leqslant\|X\|_{\left(N_{p}^{\alpha_{0}, \infty}, N_{p}^{\alpha_{1}, \infty}\right)_{\theta, q}^{R},}
\end{aligned}
$$

since $\left(N_{p}^{\alpha_{0}, q_{o}}, N_{p}^{\alpha_{1}, q_{1}}\right)_{\theta, q}^{R} \hookrightarrow\left(N_{p}^{\alpha_{0}, \infty}, N_{p}^{\alpha_{1}, \infty}\right)_{\theta, q}^{R}$, the proof is complete.

Theorem 19. Let $1<r \leqslant p<\infty, 1 \leqslant q \leqslant \infty$ and $\alpha=$ $(1 / r)-(1 / p)$ and let the filtration $G=\left\{\mathfrak{G}_{n}\right\}_{n \geqslant 1}$ be such that for every $k=1,2 \ldots$ and for all $A \in \mathfrak{G}_{k}$ the following condition holds:

$$
P(A) \geqslant \frac{C}{k},
$$

where the constant $C>0$ does not depend on $k$. Then

$$
N_{r}^{\alpha, q}(G) \hookrightarrow N_{p, q}(G)
$$


Proof. Let us show that

$$
\begin{aligned}
& N_{r}^{\alpha, 1}(G) \hookrightarrow N_{p, \infty}(G) . \\
\|X\|_{N_{p, \infty}(G)}= & \sup _{k} 2^{-k / p} \bar{X}_{2^{k}} \\
= & \sup _{k} 2^{-k / p} \sup _{A \in \mathfrak{G}_{2^{k}}} \frac{1}{P(A)}\left|\int_{A} X_{2^{k}} P(d \omega)\right| \\
\asymp & \sup _{k} 2^{-k / p} \sup _{A \in \mathfrak{G}_{2^{k}}} \frac{1}{P(A)} \\
& \times\left|\sum_{m=0}^{k} \int_{A}\left(X_{2^{m}}-X_{2^{m-1}}\right) P(d \omega)\right| \\
\leqslant & \sup _{k} \sum_{m=0}^{k} 2^{-m / p} \sup _{A \in \mathfrak{G}_{2^{m}}} \frac{1}{(P(A))^{1 / r^{\prime}}} \frac{1}{(P(A))^{1 / r}} \\
& \times\left|\int_{A}\left(X_{2^{m}}-X_{2^{m-1}}\right) P(d \omega)\right|
\end{aligned}
$$

According to the condition (80), for $A \in \mathfrak{F}_{2^{m}}$ we have that $P(A)>\left(C / 2^{m}\right)$. Therefore for $\alpha=(1 / r)-(1 / p)$ we get the following:

$$
\begin{aligned}
\|X\|_{N_{p, \infty}(G)} \leqslant & C \sup _{k} \sum_{m=0}^{k-1} 2^{-(m / p)+(m / r)} \\
& \times \sup _{A \in\left(\hat{U}_{2^{m}}\right.} \frac{1}{(P(A))^{1 / r^{\prime}}}\left|\int_{A}\left(X_{2^{m}}-X_{2^{m-1}}\right) P(d \omega)\right| \\
& \|X\|_{N_{r}^{\alpha, 1}} .
\end{aligned}
$$

Thus, (82) is proved.

Now, let $\alpha_{0}<\alpha_{1}, 1<p_{0}<p_{1}<\infty$, and $\theta \in(0,1)$ such that

$$
\begin{gathered}
\frac{1}{p_{0}}+\alpha_{0}=\frac{1}{p_{1}}+\alpha_{1}=\frac{1}{r}, \\
\alpha=(1-\theta) \alpha_{0}+\theta \alpha_{1}, \\
\frac{1}{p}=\frac{1-\theta}{p_{0}}+\frac{\theta}{p_{1}} .
\end{gathered}
$$

Then using interpolation Theorems 18 and 11 we obtain the following:

$$
\begin{gathered}
\left(N_{r}^{\alpha_{0}, 1}(G), N_{r}^{\alpha_{1}, 1}(G)\right)_{\theta, q}=N_{r}^{\alpha, q}(G), \\
\left(N_{p_{0}, \infty}(G), N_{p_{1}, \infty}(G)\right)_{\theta, q}=N_{p, q}(G) .
\end{gathered}
$$

It follows from (82) that $\left(N_{r}^{\alpha_{0}, 1}(G), N_{r}^{\alpha_{1}, 1}(G)\right)_{\theta, q} \hookrightarrow$ $\left(N_{p_{0}, \infty}(G), N_{p_{1}, \infty}(G)\right)_{\theta, q}$. Hence, $N_{r}^{\alpha, q}(G) \hookrightarrow N_{p, q}(G)$, where $\alpha=(1 / r)-(1 / p)$. The proof is complete.

\section{Spaces with Variable Approximation Properties by Haar System}

In this paragraph we consider some applications of the introduced interpolation method to Besov type spaces with variable approximation properties.

Let $\Omega=[0,1]$ and let $\mathfrak{F}$ be a $\sigma$-algebra of Borel subsets of set $\Omega, P$ a linear Lebesgue measure on $\mathfrak{F}, F=\left\{\mathfrak{F}_{n}\right\}_{n \geqslant 1}$ the Haar filtration, and $R=\left\{\tau_{k}\right\}_{k=0}^{\infty}$ a sequence of stopping times such that for any $k \geqslant 0$ the following conditions hold: $\tau_{0} \equiv 0, \tau_{k}+1 \leqslant \tau_{k+1}$ (a.p.) and

$$
\lim _{k \rightarrow \infty} \tau_{k}(\omega)=\infty \text { (a.p.). }
$$

For a function $f(x) \in L[0,1]$ we denote by $\left\{c_{k}(f)\right\}_{k \geqslant 1}$ the Fourier coefficients by Haar functions system $\left\{H_{k}(x)\right\}_{k \geqslant 1}$ ([14]). For the given stopping time $\tau_{k}(\omega)$ we denote

$$
S\left(f, \tau_{k}\right)(\omega)= \begin{cases}\sum_{m=1}^{\tau_{k}(\omega)} c_{m}(f) H_{m}(\omega) & \text { if } k \geqslant 1 \\ 0 & \text { if } k=0,\end{cases}
$$

which we call the Fourier-Haar partial sum of a function $f$, corresponding to the Markov time $\tau_{k}$.

Let $1<p<\infty, 0<q \leqslant \infty, \alpha \in \mathbb{R}$. By $B_{p}^{\alpha, q}[R]$ we denote the set of functions $f \in L[0,1]$, for which

$$
\|f\|_{B_{p}^{\alpha, q}[R]}=\left(\sum_{k=0}^{\infty} 2^{\alpha k q}\left\|f-S\left(f, \tau_{k}\right)\right\|_{L_{p}}\right)^{1 / q}<\infty
$$

for $0<q<\infty$,

$$
\|f\|_{B_{p}^{\alpha, \infty}[R]}=\sup _{k} 2^{\alpha k}\left\|f-S\left(f, \tau_{k}\right)\right\|_{L_{p}}<\infty,
$$

for $q=\infty$.

Conceptually, the introduced spaces are close to spaces with variable smoothness. Here we mention works of Leopold [15], Cobos and Fernandez [16], and Besov [17-20].

Lemma 20. Let $1<p<\infty, f \in L_{p}[0,1]$, and let $S(f, \tau)$ be the Fourier-Haar partial sum with respect to the Markov time $\tau$. Then

$$
\|S(f, \tau)\|_{p} \leqslant c\|f\|_{p}
$$

Proof. Denote

$$
\mathfrak{F}_{\tau}=\left\{A \in \mathfrak{F}: A \cap\{\tau=n\} \in \mathfrak{F}_{n} \text { for every } n \geqslant 1\right\}
$$

Let $L_{p, \infty}[0,1]$ be the Marcinkiewicz-Lorentz space. Using the equivalent norm of $L_{p, \infty}[0,1]$ spaces (see [6]) and 
martingale properties of Fourier-Haar partial sums we get the following:

$$
\begin{aligned}
& \|S(f, \tau)\|_{L_{p, \infty}[0,1]} \\
& =\sup _{A \in \mathfrak{F}, P(A)>0} \frac{1}{(P(A))^{1 / p^{\prime}}}\left|\int_{A} S(f, \tau) P(d \omega)\right| \\
& =\sup _{A \in \mathfrak{F}_{\tau}, P(A)>0} \frac{1}{(P(A))^{1 / p^{\prime}}}\left|\int_{A} S(f, \tau) P(d \omega)\right| \\
& =\sup _{A \in \mathfrak{F}_{\tau}, P(A)>0} \frac{1}{(P(A))^{1 / p^{\prime}}}\left|\int_{A} f(\omega) P(d \omega)\right| \\
& \leqslant \sup _{A \in \mathfrak{F}_{, P(A)>0}} \frac{1}{(P(A))^{1 / p^{\prime}}}\left|\int_{A} f(\omega) P(d \omega)\right| \\
& =\|f\|_{L_{p, \infty}[0,1]} .
\end{aligned}
$$

Now, applying the interpolation theorem (see [12]), we obtain the statement of the lemma.

Lemma 21. Let $1<p<\infty, 0<q \leqslant \infty, \alpha \in \mathbb{R}$, and

$$
\Delta\left(f, \tau_{k}\right)=\sum_{r=\tau_{k}+1}^{\tau_{k+1}} c_{r}(f) H_{r}(\omega) .
$$

Then

$$
\|f\|_{B_{p}^{\alpha, q}[R]}=\left(\sum_{k=0}^{\infty} 2^{\alpha k q}\left\|\Delta\left(f, \tau_{k}\right)\right\|_{L_{p}}^{q}\right)^{1 / q}
$$

Proof is similar to the proof of Lemma 3.

Theorem 22. Let $1 \leqslant q_{0}, q_{1}, q \leqslant \infty, 0<\alpha_{0}<\alpha_{1}, 0<\theta<1$, and $\alpha=(1-\theta) \alpha_{0}+\theta \alpha_{1}$. Then

$$
\left(B_{p}^{\alpha_{0}, q_{0}}[R], B_{p}^{\alpha_{1}, q_{1}}[R]\right)_{\theta, q}^{R}=B_{p}^{\alpha, q}[R] .
$$

Proof. By using Lemma 21 we have the following:

$$
\begin{aligned}
& \|f\|_{\left(B_{p}^{\alpha_{0}, q_{0}}[R], B_{p}^{\alpha_{1}, q_{1}}[R]\right)_{\theta, q}^{R}} \\
& \leqslant c\|f\|_{\left(B_{p}^{\alpha_{0}, 1}[R], B_{p}^{\alpha_{1}, 1}[R]\right)_{\theta, q}^{R}} \\
& \asymp\left(\sum _ { n = 1 } ^ { \infty } 2 ^ { \theta q n } \operatorname { i n f } _ { r } \left(\sum_{k=r}^{\infty} 2^{\alpha_{0} k}\left\|\Delta\left(f, \tau_{k}\right)\right\|_{L_{p}}\right.\right. \\
& \left.\left.+2^{-n} \sum_{k=0}^{r-1} 2^{\alpha_{1} k}\left\|\Delta\left(f, \tau_{k}\right)\right\|_{L_{p}}\right)^{q}\right)^{1 / q}
\end{aligned}
$$

$$
\begin{gathered}
\leqslant\left(\sum _ { n = 1 } ^ { \infty } 2 ^ { \theta q n } \left(\sum_{k=n \gamma}^{\infty} 2^{\alpha_{0} k}\left\|\Delta\left(f, \tau_{k}\right)\right\|_{L_{p}}\right.\right. \\
\left.\left.+2^{-n} \sum_{k=0}^{n \gamma-1} 2^{\alpha_{1} k}\left\|\Delta\left(f, \tau_{k}\right)\right\|_{L_{p}}\right)^{q}\right)^{1 / q} \\
\leqslant\left(\sum_{n=1}^{\infty}\left(2^{\theta n} \sum_{k=n \gamma}^{\infty} 2^{\alpha_{0} k}\left\|\Delta\left(f, \tau_{k}\right)\right\|_{L_{p}}\right)^{q}\right)^{1 / q} \\
+\left(\sum_{n=1}^{\infty}\left(2^{-(1-\theta) n} \sum_{k=0}^{n \gamma-1} 2^{\alpha_{1} k}\left\|\Delta\left(f, \tau_{k}\right)\right\|_{L_{p}}\right)^{q}\right)^{1 / q} .
\end{gathered}
$$

By applying Lemma 8 we get the following:

$$
\|f\|_{\left(B_{p}^{\alpha_{0}, q_{0}}[R], B_{p}^{\alpha_{1}, q_{1}}[R]\right)_{\theta, q}^{R}} \leqslant c\|f\|_{B_{p}^{\alpha, q}[R]} .
$$

Let us prove the reverse embedding. Let $f \in\left(B_{p}^{\alpha_{0}, q_{0}}[R]\right.$, $\left.B_{p}^{\alpha_{1}, q_{1}}[R]\right)_{\theta, q}^{R}, f=f_{0}+f_{1}$ be an arbitrary representation of a function, $f_{0} \in B_{p}^{\alpha_{0}, q_{0}}[R]$ and $f_{1} \in B_{p}^{\alpha_{1}, q_{1}}[R]$. Then

$$
\begin{gathered}
2^{\alpha k}\left\|\Delta\left(f, \tau_{k}\right)\right\|_{L_{p}} \\
\leqslant 2^{\alpha k}\left(\left\|\Delta\left(f_{0}, \tau_{k}\right)\right\|_{L_{p}}+\left\|\Delta\left(f_{1}, \tau_{k}\right)\right\|_{L_{p}}\right) \\
\leqslant 2^{\left(\alpha-\alpha_{0}\right) k}\left(\sup _{r \in \mathbb{N}} 2^{\alpha_{0} r}\left\|\Delta\left(f_{0}, \tau_{r}\right)\right\|_{L_{p}}\right. \\
\left.+2^{\left(\alpha_{0}-\alpha_{1}\right) k} \sup _{r \in \mathbb{N}} 2^{\alpha_{1} r}\left\|\Delta\left(f_{1}, \tau_{r}\right)\right\|_{L_{p}}\right) \\
=2^{\left(\alpha-\alpha_{0}\right) k}\left(\left\|f_{0}\right\|_{B_{p}^{\alpha_{0}, \infty}[R]}+2^{\left(\alpha_{0}-\alpha_{1}\right) k}\left\|f_{1}\right\|_{B_{p}^{\alpha_{1}, \infty}[R]}\right) .
\end{gathered}
$$

Since the representation $f=f_{0}+f_{1}$ is arbitrary, we have the following:

$$
\begin{aligned}
& 2^{\alpha k}\left\|\Delta\left(f, \tau_{k}\right)\right\|_{L_{p}} \\
& \quad \leqslant 2^{\left(\alpha-\alpha_{0}\right) k} K_{R}\left(f, 2^{\left(\alpha_{0}-\alpha_{1}\right) k} ; B_{p}^{\alpha_{0}, \infty}[R], B_{p}^{\alpha_{1}, \infty}[R]\right) .
\end{aligned}
$$

Hence, putting $a=2^{\alpha_{1}-\alpha_{0}}$ we get the following:

$$
\begin{aligned}
\|f\|_{B_{p}^{\alpha, q}[R]} & \leqslant\left(\sum_{k=0}^{\infty}\left(2^{\left(\alpha_{1}-\alpha_{0}\right) \theta k} K_{R}\left(f, 2^{\left(\alpha_{0}-\alpha_{1}\right) k}\right)\right)^{q}\right)^{1 / q} \\
& =\left(\sum_{k=0}^{\infty}\left(a^{\theta k} K_{R}\left(f, a^{-k}\right)\right)^{q}\right)^{1 / q} \\
& \leqslant\|f\|_{\left(B_{p}^{\alpha_{0}, \infty}[R], B_{p}^{\alpha_{1}, \infty}[R]\right)_{\theta, q}^{R}} \\
& \leqslant c\|f\|_{\left(B_{p}^{\alpha_{0}, q_{0}}[R], B_{p}^{\alpha_{1}, q_{1}}[R]\right]_{\theta, q}^{R} .}
\end{aligned}
$$

The theorem is proved. 


\section{Acknowledgment}

This research was partially supported by Ministry of Education and Science of the Republic of Kazakhstan (0112RK02176, 0112RK00608).

\section{References}

[1] D. L. Burkholder and R. F. Gundy, "Extrapolation and interpolation of quasi-linear operators on martingales," Acta Mathematica, vol. 124, pp. 249-304, 1970.

[2] J.-A. Chao and R.-L. Long, "Martingale transforms with unbounded multipliers," Proceedings of the American Mathematical Society, vol. 114, no. 3, pp. 831-838, 1992.

[3] J.-A. Chao and R.-L. Long, "Martingale transforms with unbounded multipliers," Transactions of the American Mathematical Society, vol. 193, no. 3, pp. 199-215, 1974.

[4] F. Weisz, "Hardy spaces of predictable martingales," Analysis Mathematica, vol. 20, no. 3, pp. 225-233, 1994.

[5] F. Weisz, "Weak martingale Hardy spaces," Probability and Mathematical Statistics, vol. 18, no. 1, pp. 133-148, Acta Universitatis Wratislaviensis, no. 2045, pp. 133-148, 1998.

[6] E. D. Nursultanov, "Network spaces and inequalities of HardyLittlewood type," Matematicheskiu Sbornik, vol. 189, no. 3, pp. 83-102 (Russian), Translation in Sbornik: Mathematics, vol. 189, no. 3, pp. 399-419, 1998.

[7] E. D. Nursultanov, "Interpolation properties of some anisotropic spaces and Hardy-Littlewood type inequalities," East Journal on Approximations, vol. 4, no. 2, pp. 243-275, 1998.

[8] E. D. Nursultanov and S. Tikhonov, "Net spaces and boundedness of integral operators," Journal of Geometric Analysis, vol. 21, no. 4, pp. 950-981, 2011.

[9] A. N. Shiryaev, Probability, Nauka, Moscow, Russia, 1980, (Russian).

[10] T. U. Aubakirov and E. D. Nursultanov, "Spaces of stochastic processes, and interpolation theorems," Uspekhi Matematicheskikh Nauk, vol. 61, no. 6, pp. 181-182 (Russian), Translation in Russian Mathematical Surveys, vol. 61, no. 6, pp. 1167-1169, 2006.

[11] J. L. Doob, Stochastic Processes, Wiley Classics Library, John Wiley \& Sons Inc., New York, NY, USA, 1990.

[12] J. Bergh and J. Löfström, Interpolation Spaces: An Introduction, Grundlehren der Mathematischen Wissenschaften, no. 223, Springer, Berlin, Germany, 1976.

[13] T. U. Aubakirov and E. D. Nursultanov, "Interpolation theorem for stochastic processes," Eurasian Mathematical Journal, vol. 1, no. 1, pp. 8-16, 2010.

[14] B. S. Kashin and A. A. Saakyan, Orthogonal Series, Nauka, Moscow, Russia, 1984.

[15] H.-G. Leopold, "On function spaces of variable order of differentiation," Forum Mathematicum, vol. 3, no. 1, pp. 1-21, 1991.

[16] F. Cobos and D. L. Fernandez, "Hardy-Sobolev spaces and Besov spaces with a function parameter," in Function Spaces and Applications, vol. 1302 of Lecture Notes in Mathematics, pp. 158170, Springer, Berlin, Germany, 1988.

[17] O. V. Besov, "Interpolation and embeddings of the spaces of generalized functions spaces $B_{p, q}^{s}$ and $F_{p, q}^{s}$ on a domain," Trudy Matematicheskogo Instituta Imeni V. A. Steklova, vol. 219, pp. 80102 (Russian), Translation in Proceedings of the Steklov Institute of Mathematics, no. 4, pp. 73-95, 1997.
[18] O. V. Besov, "On spaces of functions of variable smoothness defined by pseudodifferential operators," Trudy Matematicheskogo Instituta Imeni V. A. Steklova, vol. 227, pp. 56-74 (Russian), Translation in Proceedings of the Steklov Institute of Mathematics, no. 4, pp. 50-69, 1999.

[19] O. V. Besov, "Equivalent normings of spaces of functions of variable smoothness," Trudy Matematicheskogo Instituta Imeni V. A. Steklova, vol. 243, pp. 87-95 (Russian), Translation in Proceedings of the Steklov Institute of Mathematics, no. 4, pp. 8088, 2003.

[20] O. V. Besov, "On the interpolation, embedding, and extension of spaces of functions of variable smoothness," Doklady Akademii Nauk, vol. 401, no. 1, pp. 7-11, 2005 (Russian). 


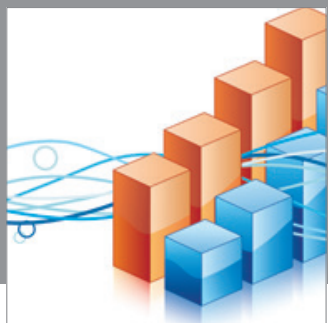

Advances in

Operations Research

mansans

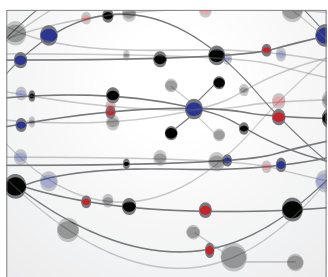

The Scientific World Journal
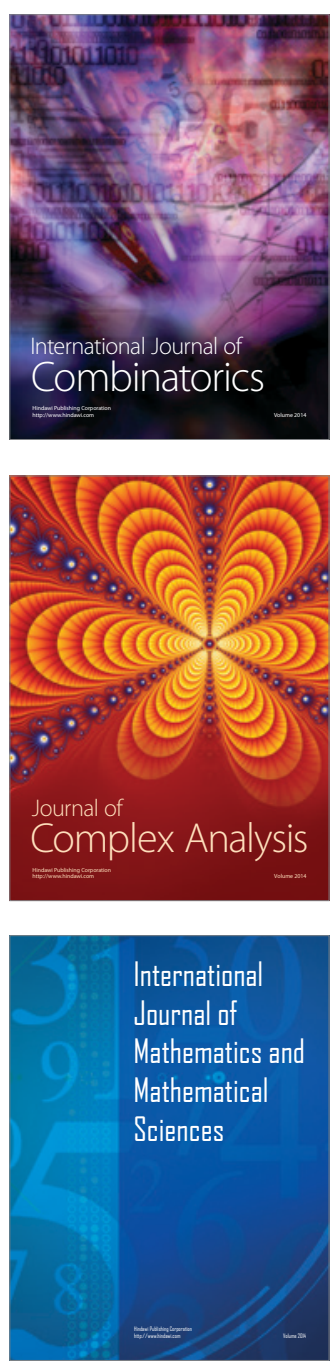
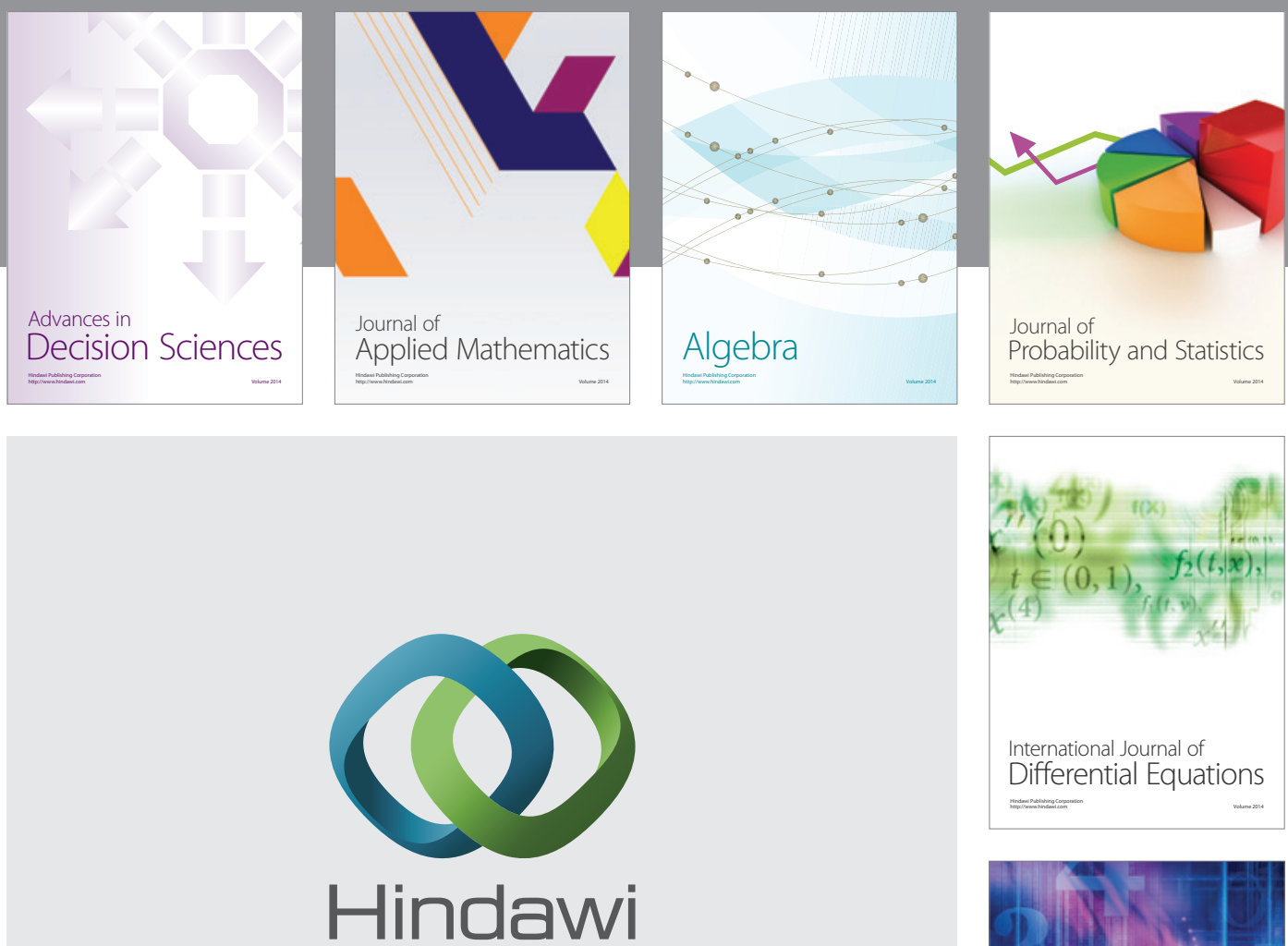

Submit your manuscripts at http://www.hindawi.com
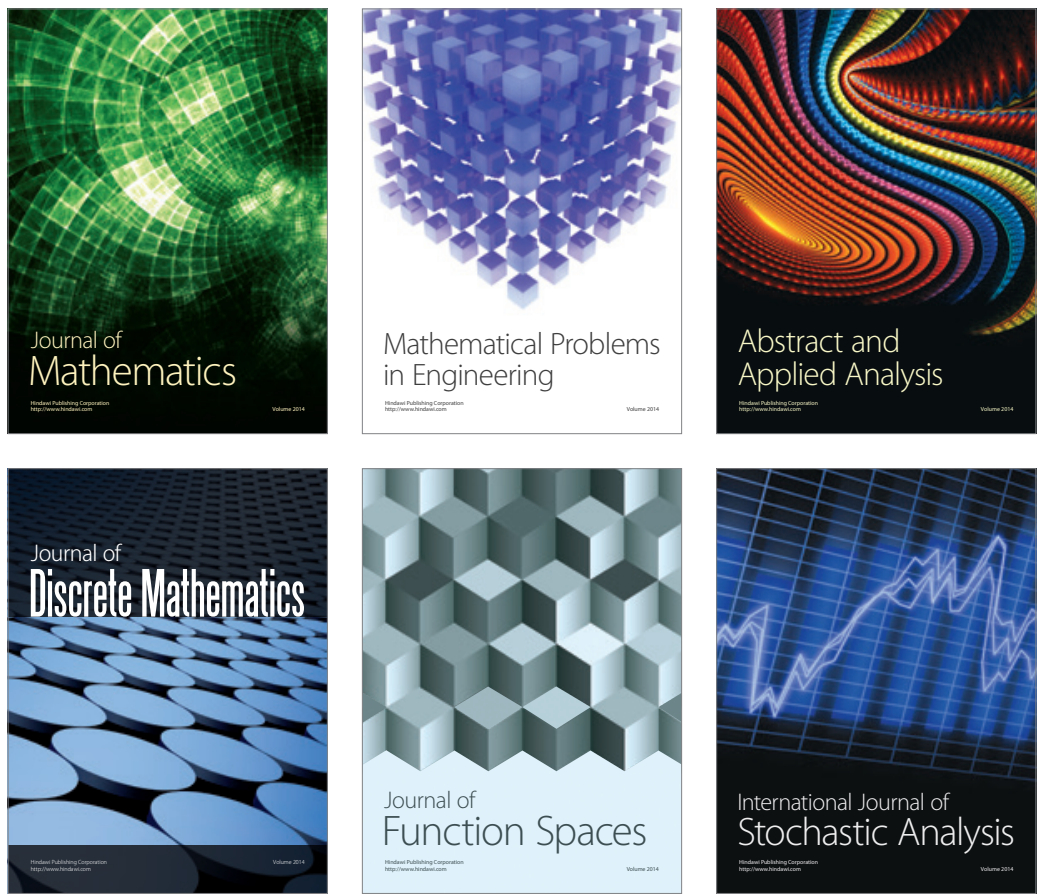

Journal of

Function Spaces

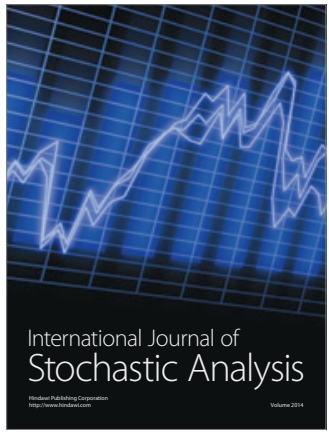

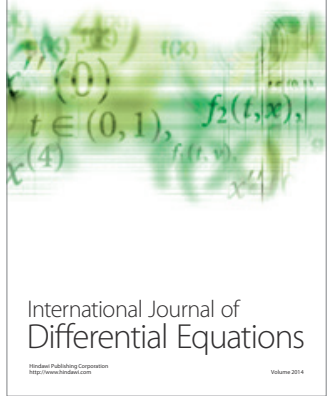
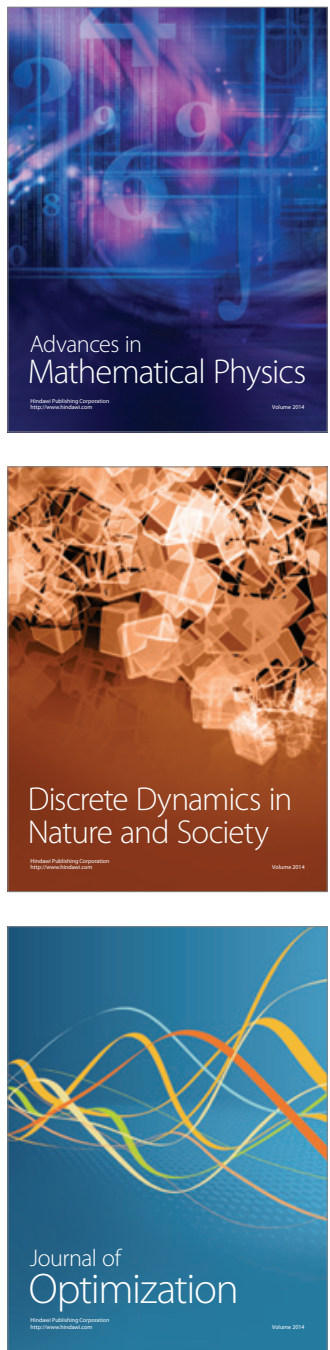